\title{
Caracterização dos casos notificados de violência interpessoal e autoprovocada
}

\section{Characterization of notified cases of interpersonal self-protected violence}

\section{Caracterización de los casos notificados de violencia interpersonal autoprovocada}

\section{Recebido: 22/06/2017 \\ Aprovado: 21/11/2018 Publicado: 29/01/2019}

\author{
Ana Lígia Fernandes Reis ${ }^{1}$ \\ Sônia Aparecida da Cruz Oliveira ${ }^{2}$ \\ Gabriela Martins Espolador ${ }^{3}$ \\ Alexandre Lins Werneck ${ }^{4}$
}

0 artigo teve como objetivo caracterizar casos de notificações de violência. Este é um estudo quantitativo, com delineamento descritivo, de corte transversal, realizado com dados do Sistema de Informações de Agravos de Notificação, considerando dados do ano de 2015 da cidade de São José do Rio Preto, SP. Foram elaboradas tabelas considerando-se padrão de análise de significância de $\mathrm{p}<0,05$, com nível de confiança de $95 \%$. Houveram registros de 1.698 casos, das quais $71,2 \%$ no sexo feminino. A violência física acometeu $54,4 \%$ e a negligenciada $34,3 \%$. A idade de 0 - 9 anos representaram 81,9\% da negligenciada, com maior ocorrência em 69\% na residência. A mãe, seguida do pai foram os principais agressores nas idades de $0-9$ anos $(68,8 \%)$ e $(31,4 \%)$ e, de $10-19$ anos $(28,4 \%)$ e (15\%). Alto grau de incidência da própria pessoa - autoprovocada (67,9\%), (20-39-40) anos ou mais. Encaminhados para o Conselho Tutelar nas faixas etárias de 0-9 anos (84,2\%), e 10-19 anos (61,8\%), e entre 2039 anos ao atendimento à mulher e Delegacia da Mulher em 44\% e 52\% respectivamente. A pesquisa mostrou a necessária articulação e organização entre as redes de apoio e mais pesquisas destacando as questões das violências por ciclos de vida para elaboração de ações de prevenção e controle.

Descritores: Notificação; Violência doméstica; Maus-tratos Infantis; Violência contra a mulher; Maus-tratos ao idoso.

This article aimed to characterize the reported cases of violence. This is a descriptive, cross-sectional study with a quantitative approach, carried out with data from the Information System of Health Problem Notifications, considering data from 2015, from the city of São José do Rio Preto, SP, Brazil. Tables were formulated considering as significant results of $\mathrm{p}<0.05$, with a confidence level of $95 \%$. There were 1,698 cases, from which $71.2 \%$ of the victims were female. Physical violence affected $54.4 \%$ and negligence $34.3 \%$. People from 0 to 9 years of age were the victims in $81.9 \%$ of negligence cases, and $69 \%$ of them occurred at home. The mother, followed by the father, were the main aggressors in victims who were $0-9$ years $(68.8 \%$ and $31.4 \%)$ and $10-19$ years $(28.4 \%$ and $15 \%)$. A high incidence of self- inflicted violence was found (67.9\%), (20-39-40) years or more. $84.2 \%$ and $61.8 \%$ of the 0-9 and of 10-19 age groups were sent to the Child Protection Agency, respectively. Between the ages of 20 and 39 , the number of women attending a specialized service and the Women's Police Station was 44\% and 52\%, respectively. The research showed the articulation and organization that must exist between the support networks and other researches that highlight the issues of violence in each age group, for the elaboration of preventive and control actions.

Descriptors: Notification; Domestic violence; Child abuse; Violence against women; Elder abuse.

El artículo tuvo como objetivo caracterizar casos de notificaciones de violencia. Este es un estudio cuantitativo, con delineamiento descriptivo, de corte transversal, realizado con datos del Sistema de Informaciones de Agravamientos de Notificación, considerando datos del año 2015 de la ciudad de São José do Rio Preto, SP, Brasil. Fueron elaboradas tablas considerándose estándar de análisis de significancia de p<0,05 con nivel de confianza de 95\%. Hubo registros de 1.698 casos, de los cuales 71,2\% eran del sexo femenino. La violencia física afectó 54,4\% y la de descuido 34,3\%. La edad de 0-9 años representó 81,9\% de la violencia de descuido, con mayor aparición en $69 \%$ en la residencia. La madre, seguida del padre fueron los principales agresores en las edades de 0-9 años $(68,8 \%)$ y $(31,4 \%)$ y, de $10-19$ años $(28,4 \%)$ y (15\%). Alto grado de incidencia de la propia persona - auto provocada 67,9\%), (20-39-40) años o más. Derivados al Consejo Tutelar en los grupos etarios de 0-9 años (84,2\%), y 10-19 años (61,8\%), y entre 20-39 años al atendimiento a la mujer y Comisaría de la Mujer en 44\% y 52\% respectivamente. La investigación mostró la necesaria articulación y organización entre las redes de apoyo y más investigaciones destacando las cuestiones de las violencias por ciclos de vida para la elaboración de acciones de prevención y control.

Descriptores: Notificación; Violencia doméstica; Maltrato ao los niños; Violencia contra la mujer; Maltrato al anciano.

1. Enfermeira. Especialista em Gestão de Saúde. Especialista em Atenção Básica com ênfase na Estratégia de Saúde da Família, São José do Rio Preto, SP, Brasil. ORCID: 0000-0002-6408-5897 E-mail: ana-ligia17@hotmail.com

2. Enfermeira. Especialista em Administração Hospitalar. Mestre em Enfermagem em Saúde Pública. Professora do Curso de Enfermagem da Faculdade de Medicina de São José do Rio Preto (FAMERP), SP, Brasil. ORCID: 0000-0003-2346-8514 E-mail: soniaoliveira@famerp.br

3. Enfermeira. Especialista em Atenção Básica com ênfase na Estratégia de Saúde da Família. Professora Substituta da Faculdade de Mirassol, SP, Brasil. ORCID: 0000-0001-7644-6859 E-mail: gespolador@gmail.com

4. Bacharel em Letras. Mestre e Doutor em Ciências da Saúde. Professor do Programa de Pós Graduação em Enfermagem da FAMERP, São José do Rio Preto, SP, Brasil. ORCID: 0000-0002-2911-8091 E-mail: alexandre.werneck@famerp.br 


\section{INTRODUÇÃO}

$\mathrm{A}$ violência tem sido apontada como relevante problema de saúde pública, existente em qualquer meio social ${ }^{1}$. Embora seja de essência obscura, um dos conceitos aplicados a reconhece como o uso da força física ou da autoridade, em coação ou ação, autoprovocada ou dirigida à outra pessoa ou grupo social, que resulte, ou não em lesão, óbito, dano moral, desenvolvimento prejudicado ou privação ${ }^{2}$.

No cenário mundial a violência é um dos principais motivos de óbito na faixa etária de 15 a 44 anos. E são notórios os índices de violência doméstica como maior causa de lesões e mortes de mulheres entre 14 a 44 anos$^{1}$. Em 2012, no Brasil, as mortes por causas externas totalizaram 152.013, o que retrata $12,9 \%$ de todas as causas de morte no país. Parcela significativa dessas mortes está caracterizada por violências das quais: $37 \%$ são agressões, que adicionadas às lesões autoprovocadas e às intervenções legais englobam $44,3 \%$ de todas as mortes por causas externas, demonstrando a frequente adversidade que torna vulnerável a população brasileira ${ }^{3}$.

Analisando o grande impacto social e econômico exercido pela violência, principalmente no setor saúde e, considerando que estratégias embasadas na vigilância, prevenção e promoção da saúde são essenciais para a tentativa de resolutividade desse revés, em 2006 o Ministério da Saúde, por meio de sua Secretaria de Vigilância em Saúde (SVS), instituiu o Sistema de Vigilância contínua de violência doméstica, sexual e outras violências interpessoais do Sistema de Informação de Agravos de Notificação (VIVA/SINAN) 1,3,4.

Por meio do Sistema de Informação de Agravos de Notificação (SINAN), a notificação é compulsória e, fundamenta-se em casos de violência relacionadas com crianças, adolescentes, mulheres, idosos e, população LGBT $^{4}$. Segundo o que está estabelecido no Estatuto da Criança e do Adolescente, pelas Leis de $\mathrm{n}^{\mathrm{o}} 8.069 / 1990^{5}$, na Lei $\mathrm{n}^{\mathrm{o}}$ $10.778 / 2003$ que estabelece a Notificação de Violência contra a Mulher6, no Estatuto do Idoso pela Lei no $10.741 / 2003^{7}$ e na portaria no 2.836/2011 que institui notificação compulsória contra população LGBT .

A vigilância epidemiológica de violências se propõe, além de coletar dados que possibilitem descrever os casos desses agravos, anseia fundamentar ações, bem como, amparar a formação de redes de apoio às pessoas vitimizadas pela violência $e$, garantir atendimento integral, promoção da saúde e cultura de paz ${ }^{4}$.

Embora haja leis no combate à violência, no Brasil, há grandes lacunas na rede de apoio as vítimas. Portanto, caracterizar os casos de violência e mostrar as causas influenciadoras, pode auxiliar discussões no aprimoramento das políticas públicas e organização da rede de apoio, além de aguçar a análise da sociedade sobre a relevância do combate à violência.

São inúmeras as notícias sobre violência nos telejornais, diariamente, e com a crise política e econômica em que o país se encontra, aumenta ainda mais esse drama, sem correlação com a classe social e a idade dos que são agredidos e dos que praticam a violência autoprovocada.

Diante desse contexto, da relevância do tema, este estudo tem como proposta caracterizar os casos de notificações de violência, para corroborar ações de vigilância em saúde e realizar planejamento estratégico para promoção, prevenção e controle dessas violências, em conjunto com as redes de apoio. Desta forma, este estudo teve como objetivo caracterizar os casos de notificações de violência.

\section{MÉTODO}

Trata-se de estudo quantitativo, com delineamento descritivo, de corte transversal, utilizando dados secundários, a partir do banco de dados do SINAN da Secretaria Municipal de Saúde (SMS), da cidade de São José do Rio Preto - SP, com informações das fichas de notificação de violência doméstica, sexual e outras violências interpessoais, no período de janeiro a dezembro de 2015.

As variáveis estudadas foram classificadas em relação à vítima, aos tipos de violência, em relação ao provável agressor e em relação ao encaminhamento das vitimas para os serviços de apoio. Isto, conforme 
registro feito pelo profissional de saúde que atendeu o caso suspeito ou confirmado de violência. Foram analisadas as seguintes variáveis:

a) Caracterização das vítimas de violência: sexo (masculino e feminino); idade (categorizada nas faixas etárias de 0 a 9, 10 a 19, 20 a 39, 40 anos ou mais); cor da pele ou raça (branca, preta, outras). Escolaridade (analfabeto, ensino fundamental incompleto e ensino fundamental completo, outros) local de ocorrência (residência e outros) e quantas vezes a vitima foi violentada (se apenas uma vez ou mais de uma vez);

b) Tipos de violência: casos suspeitos ou confirmados de lesão autoprovocada; violência doméstica de natureza física, psicológica, sexual, negligência; violência infantil e, outras violências;

c) Vínculo da vítima com o provável agressor: pai, mãe, cônjuge, própria pessoa, outras pessoas;

d) Encaminhamentos: se as vítimas de violência foram encaminhadas para os serviços de apoio do Município: rede de saúde, rede de assistência social, rede de atendimento a mulher, conselho tutelar e delegacia da mulher.

A partir da coleta de dados amostrais, criou-se um banco de dados contemplando 1.698 registros, subdivididos entre 74 variáveis que abordam dados clínicos e sociodemográficos. Após, foram selecionadas algumas variáveis pertinentes para esse trabalho (anteriormente descritas) e foram criados extratos de idade que seguiram a classificação: 0-9 anos, 10 a 19 anos, 20 a 39 anos, 40 anos ou mais.

Após essa etapa, as informações foram submetidas a dois tipos de análises, sendo elas: descritiva e inferencial. No âmbito descritivo, os dados coletados foram expostos por meio de valores consolidados $(\mathrm{N})$ e as respectivas proporções (\%). Esses dados foram subdivididos entre tabelas elaboradas em programa Excel e editadas pelo software Microsoft Word ${ }^{\circledR}$ (2.016).

$\mathrm{Na}$ parte inferencial da análise, foi aplicado o teste regressão linear, que analisa a existência de dependência estatística significante entre as variáveis analisadas. Essa validação é feita pela análise do valor de (p), com a seguinte conclusão: $p<0,05$ existe evidência de dependência estatística entre as vaiáveis analisadas. E, para todos os cruzamentos aplicados, considerou-se o nível de confiança de 95\%.

Esta pesquisa seguiu as normas do Conselho Nacional de Saúde 466/12, sendo respeitados, todos os aspectos éticos, legalmente previstos. Os dados foram utilizados de forma sigilosa e em conjunto, sem individualização ou identificação de participantes da pesquisa, com aprovação no Comitê de Ética e Pesquisa da Faculdade de Medicina de São José do Rio Preto, sob o parecer $\mathrm{n}$ 을.723.984.

\section{RESULTADOS}

Foram registradas 1.698 notificações de violência, das quais $71 \%$ referiam-se a vitimas do sexo feminino e $29 \%$ ao sexo masculino. As maiores proporções de casos notificados foram identificadas entre adultos jovens $(20 \mathrm{a}$ 39 anos), apresentando distribuições diferentes quando analisadas entre os sexos. Para os homens, a faixa etária mais acometida foi a de 0 a 9 anos em $52 \%$, seguida da de 10 a 19 anos com 29,4\%. Entre as mulheres, a maior proporção de ocorrência de violência por faixa de idade foi observada naquelas de 20 a 39 anos com $84,7 \%$ e, de 10 a 19 anos com $70,6 \%$.

A violência contra o sexo feminino se deu em $71 \%$ dos casos e (29\%) contra o sexo masculino. Pela análise inferencial aplicada ao modelo de regressão linear se encontrou um valor de $(p=0,00)$, se posicionando a esquerda do valor de alfa-padrão, mostrando tendência à dependência estatística entre as variáveis, faixa etária e sexo.

No que se referem à raça/cor, os que se declaram brancos representaram 71\%, seguidos de outros em 17,4\%. Quanto à escolaridade outros (aqui representados por ensino médio completo e ensino superior completo e incompleto) tem-se 60,9\%, seguido do ensino fundamental incompleto em $39,1 \%$ na faixa etária de 10 a 19 anos.

A residência foi o local onde mais ocorreu a violência em $68,9 \%$ e $36,8 \%$ dos indivíduos sofreram violência mais de uma vez. 
Para ambas variáveis analisadas (raça/cor e dependência quando cruzado com a variável escolaridade, o comportamento do valor de $\mathrm{p}$ resposta faixa etária. Conforme Tabela 1.

foram idênticos $p=0,00$ ), também

comprovando a tendência estatística de

Tabela 1. Caracterização sociodemográfica, local de ocorrência e número de violências sofridas. São José do Rio Preto, SP, 2015.

\begin{tabular}{|c|c|c|c|c|c|c|c|c|c|c|c|}
\hline \multirow{2}{*}{$\begin{array}{c}\text { Informação } \\
\text { Sexo }\end{array}$} & \multicolumn{2}{|c|}{$\begin{array}{l}0 \text { a } 9 \\
\text { anos }\end{array}$} & \multicolumn{2}{|c|}{$\begin{array}{c}10 \text { a } 19 \\
\text { anos }\end{array}$} & \multicolumn{2}{|c|}{$\begin{array}{c}20 \text { a } 39 \\
\text { anos }\end{array}$} & \multicolumn{2}{|c|}{$\begin{array}{c}40 \text { anos ou } \\
\text { mais }\end{array}$} & \multicolumn{2}{|c|}{ Total } & \multirow[t]{2}{*}{$\begin{array}{c}\text { Valor } \\
\text { p }\end{array}$} \\
\hline & $\mathbf{N}$ & $\%$ & $\mathbf{N}$ & $\%$ & $\mathbf{N}$ & $\%$ & $\mathbf{N}$ & $\%$ & $\mathbf{N}$ & $\%$ & \\
\hline Feminino & 209 & 47,3 & 231 & 70,6 & 460 & 84,7 & 306 & 79,3 & 1206 & 71,0 & \multirow[b]{3}{*}{$\mathbf{0 , 0 0 0}$} \\
\hline Masculino & 233 & 52,7 & 96 & 29,4 & 83 & 15,3 & 80 & 20,7 & 492 & 29,0 & \\
\hline TOTAL & 442 & 100,0 & 327 & 100,0 & 543 & 100,0 & 386 & 100,0 & 1698 & 100,0 & \\
\hline Raça & $\mathbf{N}$ & $\%$ & $\mathbf{N}$ & $\%$ & $\mathbf{N}$ & $\%$ & $\mathbf{N}$ & $\%$ & $\mathbf{N}$ & $\%$ & \\
\hline Branca & 288 & 65,2 & 215 & 65,7 & 403 & 74,2 & 298 & 77,2 & 1204 & 70,9 & \multirow[b]{5}{*}{0,000} \\
\hline Preta & 20 & 4,5 & 16 & 4,9 & 45 & 8,3 & 27 & 7,0 & 108 & 6,4 & \\
\hline Outras & 81 & 18,3 & 80 & 24,5 & 86 & 15,8 & 48 & 12,4 & 295 & 17,4 & \\
\hline Ignorado & 53 & 12,0 & 16 & 4,9 & 9 & 1,7 & 13 & 3,4 & 91 & 5,4 & \\
\hline TOTAL & 442 & 100,0 & 327 & 100,0 & 543 & 100,0 & 386 & 100,0 & 1698 & 100,0 & \\
\hline Escolaridade & $\mathbf{N}$ & $\%$ & $\mathbf{N}$ & $\%$ & $\mathbf{N}$ & $\%$ & $\mathbf{N}$ & $\%$ & $\mathbf{N}$ & $\%$ & \\
\hline Analfabeto & 1 & 0,2 & 1 & 0,3 & 1 & 0,2 & 5 & 1,3 & 8 & 0,5 & \multirow[b]{6}{*}{0,000} \\
\hline Ens. Fund. Incomp. & 3 & 0,7 & 128 & 39,1 & 71 & 13,1 & 54 & 14,0 & 256 & 15,1 & \\
\hline Ens. Fund. Comp. & 0 & 0,0 & 7 & 2,1 & 33 & 6,1 & 19 & 4,9 & 59 & 3,5 & \\
\hline Outros & 419 & 94,8 & 120 & 36,7 & 322 & 59,3 & 173 & 44,8 & 1034 & 60,9 & \\
\hline Ignorado & 19 & 4,3 & 71 & 21,7 & 116 & 21,4 & 135 & 35,0 & 341 & 20,1 & \\
\hline TOTAL & 442 & 100,0 & 327 & 100,0 & 543 & 100,0 & 386 & 100,0 & 1698 & 100,0 & \\
\hline $\begin{array}{c}\text { Local da } \\
\text { Ocorrência }\end{array}$ & $\mathbf{N}$ & $\%$ & $\mathbf{N}$ & $\%$ & $\mathbf{N}$ & $\%$ & $\mathbf{N}$ & $\%$ & $\mathbf{N}$ & $\%$ & \\
\hline Residência & 241 & 54,5 & 189 & 57,8 & 423 & 77,9 & 317 & 82,1 & 1170 & 68,9 & \multirow[b]{3}{*}{0,049} \\
\hline Ignorado & 18 & 4,1 & 16 & 4,9 & 22 & 4,1 & 8 & 2,1 & 64 & 3,8 & \\
\hline TOTAL & 442 & 100,0 & 327 & 100,0 & 543 & 100,0 & 386 & 100,0 & 1698 & 100,0 & \\
\hline Outras Vezes & $\mathbf{N}$ & $\%$ & $\mathbf{N}$ & $\%$ & $\mathbf{N}$ & $\%$ & $\mathbf{N}$ & $\%$ & $\mathbf{N}$ & $\%$ & \\
\hline Sim & 83 & 18,8 & 95 & 29,1 & 259 & 47,7 & 188 & 48,7 & 625 & 36,8 & \\
\hline Não & 177 & 40,0 & 153 & 46,8 & 222 & 40,9 & 115 & 29,8 & 667 & 39,3 & \\
\hline Ignorado & 182 & 41,2 & 79 & 24,2 & 62 & 11,4 & 83 & 21,5 & 406 & 23,9 & \\
\hline TOTAL & 442 & 100,0 & 327 & 100,0 & 543 & 100,0 & 386 & 100,0 & 1698 & 100,0 & 0,001 \\
\hline
\end{tabular}

A forma de violência mais notificada foi à física em $54,4 \%$, seguida pela negligenciada em $34,3 \%$ e, em terceiro lugar a violência psicológica e lesão autoprovocada apresentaram a mesma frequência de ocorrência $(24,6 \%)$. As violências foram mais frequentes na faixa etária de 20 a 39 anos, com exceção da violência por negligência, mais prevalente na faixa etárias de 0 a 9 anos (81,9\%), seguido da violência física $(18,6 \%)$.
Estes resultados demonstram que ainda as crianças e adolescentes estão sendo vítimas de negligências e ou abandonos pelos seus pais e ou cuidadores $(\mathrm{p}=0,00)$. (Tabela 2$)$.

Quanto aos perpetradores da violência, a mãe seguida do pai foram os principais agressores nas faixas etárias de $0-9$ anos $(68,8 \%$ e $31,4 \%$, respectivamente) e de 10 a 19 anos $(28,4 \%$ e $15 \%$, respectivamente). 
Tabela 2. Violência por faixa etária e tipos. São José do Rio Preto, SP, 2015.

\begin{tabular}{|c|c|c|c|c|c|c|c|c|c|c|c|}
\hline \multirow{2}{*}{$\begin{array}{c}\text { Informação } \\
\text { Lesão } \\
\text { Autoprovocada }\end{array}$} & \multicolumn{2}{|c|}{$\begin{array}{l}0 \text { a } 9 \\
\text { anos }\end{array}$} & \multicolumn{2}{|c|}{10 a 19 anos } & \multicolumn{2}{|c|}{20 a 39 anos } & \multicolumn{2}{|c|}{$\begin{array}{c}40 \text { anos ou } \\
\text { mais }\end{array}$} & \multicolumn{2}{|c|}{ Total } & \multirow[t]{2}{*}{$\begin{array}{c}\text { Valor } \\
\mathbf{P} \\
\end{array}$} \\
\hline & $\mathbf{N}$ & $\%$ & $\mathbf{N}$ & $\%$ & $\mathbf{N}$ & $\%$ & $\mathbf{N}$ & $\%$ & $\mathbf{N}$ & $\%$ & \\
\hline Sim & 2 & 0,5 & 95 & 29,1 & 199 & 36,6 & 121 & 31,3 & 417 & 24,6 & \\
\hline Não & 409 & 92,5 & 198 & 60,6 & 324 & 59,7 & 240 & 62,2 & 1171 & 69,0 & \\
\hline Ignorado & 31 & 7,0 & 34 & 10,4 & 20 & 3,7 & 25 & 6,5 & 110 & 6,5 & \\
\hline TOTAL & 442 & 100,0 & 327 & 100,0 & 543 & 100,0 & 386 & 100,0 & 1698 & 100,0 & 0,817 \\
\hline Violência Física & $\mathbf{N}$ & $\%$ & $\mathbf{N}$ & $\%$ & $\mathbf{N}$ & $\%$ & $\mathbf{N}$ & $\%$ & $\mathbf{N}$ & $\%$ & \\
\hline Sim & 82 & 18,6 & 162 & 49,5 & 443 & 81,6 & 237 & 61,4 & 924 & 54,4 & \\
\hline Não & 349 & 79,0 & 160 & 48,9 & 100 & 18,4 & 141 & 36,5 & 750 & 44,2 & \\
\hline Ignorado & 11 & 2,5 & 5 & 1,5 & 0 & 0,0 & 8 & 2,1 & 24 & 1,4 & \\
\hline TOTAL & 442 & 100,0 & 327 & 100,0 & 543 & 100,0 & 386 & 100,0 & 1698 & 100,0 & 0,000 \\
\hline $\begin{array}{l}\text { Violência } \\
\text { Psicológica }\end{array}$ & $\mathbf{N}$ & $\%$ & $\mathbf{N}$ & $\%$ & $\mathbf{N}$ & $\%$ & $\mathbf{N}$ & $\%$ & $\mathbf{N}$ & $\%$ & \\
\hline Sim & 41 & 9,3 & 61 & 18,7 & 205 & 37,8 & 110 & 28,5 & 417 & 24,6 & \\
\hline Não & 391 & 88,5 & 258 & 78,9 & 338 & 62,2 & 264 & 68,4 & 1251 & 73,7 & \\
\hline Ignorado & 10 & 2,3 & 8 & 2,4 & 0 & 0,0 & 12 & 3,1 & 30 & 1,8 & \\
\hline TOTAL & 442 & 100,0 & 327 & 100,0 & 543 & 100,0 & 386 & 100,0 & 1698 & 100,0 & 0,227 \\
\hline Violência Sexual & $\mathbf{N}$ & $\%$ & $\mathbf{N}$ & $\%$ & $\mathbf{N}$ & $\%$ & $\mathbf{N}$ & $\%$ & $\mathbf{N}$ & $\%$ & \\
\hline Sim & 52 & 11,8 & 63 & 19,3 & 43 & 7,9 & 10 & 2,6 & 168 & 9,9 & \\
\hline Não & 380 & 86,0 & 255 & 78,0 & 497 & 91,5 & 369 & 95,6 & 1501 & 88,4 & \\
\hline Ignorado & 10 & 2,3 & 9 & 2,8 & 3 & 0,6 & 7 & 1,8 & 29 & 1,7 & \\
\hline TOTAL & 442 & 100,0 & 327 & 100,0 & 543 & 100,0 & 386 & 100,0 & 1698 & 100,0 & 0,550 \\
\hline $\begin{array}{c}\text { Violência } \\
\text { Negligenciada }\end{array}$ & $\mathbf{N}$ & $\%$ & $\mathbf{N}$ & $\%$ & $\mathbf{N}$ & $\%$ & $\mathbf{N}$ & $\%$ & $\mathbf{N}$ & $\%$ & \\
\hline Sim & 362 & 81,9 & 113 & 34,6 & 7 & 1,3 & 100 & 25,9 & 582 & 34,3 & \\
\hline Não & 78 & 17,6 & 206 & 63,0 & 533 & 98,2 & 280 & 72,5 & 1097 & 64,6 & \\
\hline Ignorado & 2 & 0,5 & 8 & 2,4 & 3 & 0,6 & 6 & 1,6 & 19 & 1,1 & \\
\hline TOTAL & 442 & 100,0 & 327 & 100,0 & 543 & 100,0 & 386 & 100,0 & 1698 & 100,0 & 0,000 \\
\hline $\begin{array}{l}\text { Violência } \\
\text { Infantil }\end{array}$ & $\mathbf{N}$ & $\%$ & $\mathbf{N}$ & $\%$ & $\mathbf{N}$ & $\%$ & $\mathbf{N}$ & $\%$ & $\mathbf{N}$ & $\%$ & \\
\hline Sim & 2 & 0,5 & 2 & 0,6 & 0 & 0,0 & 0 & 0,0 & 4 & 0,2 & \\
\hline Não & 430 & 97,3 & 316 & 96,6 & 540 & 99,4 & 379 & 98,2 & 1665 & 98,1 & \\
\hline Ignorado & 10 & 2,3 & 9 & 2,8 & 3 & 0,6 & 7 & 1,8 & 29 & 1,7 & \\
\hline TOTAL & 442 & 100,0 & 327 & 100,0 & 543 & 100,0 & 386 & 100,0 & 1698 & 100,0 & 0,006 \\
\hline $\begin{array}{c}\text { Outras } \\
\text { Violências }\end{array}$ & $\mathbf{N}$ & $\%$ & $\mathbf{N}$ & $\%$ & $\mathbf{N}$ & $\%$ & $\mathbf{N}$ & $\%$ & $\mathbf{N}$ & $\%$ & \\
\hline Sim & 25 & 5,7 & 37 & 11,3 & 102 & 18,8 & 53 & 13,7 & 217 & 12,8 & \\
\hline Não & 403 & 91,2 & 275 & 84,1 & 435 & 80,1 & 323 & 83,7 & 1436 & 84,6 & \\
\hline Ignorado & 14 & 3,2 & 15 & 4,6 & 6 & 1,1 & 10 & 2,6 & 45 & 2,7 & \\
\hline TOTAL & 442 & 100,0 & 327 & 100,0 & 543 & 100,0 & 386 & 100,0 & 1698 & 100,0 & 0,047 \\
\hline
\end{tabular}

Notou-se alto grau de incidência da própria pessoa, como principal autor da agressão, nas faixas etárias de 20 a 39 anos $(37,8 \%)$ e de 40 anos ou mais (32,9\%), seguida a faixa etária de 10 a 19 anos de idade $(27,2 \%)$.

Um familiar era o provável autor da agressão, e a mãe apareceu em primeiro lugar seguido do pai e depois o cônjuge na faixa etária de 20 a 39 anos $(25,4 \%)$ e de 40 anos ou mais $(14,8 \%)$. Nesse caso, o cruzamento entre os registros de violência partindo do Cônjuge, ao ser cruzado com os dados de Faixa Etária de acordo com os dados coletados, aponta-se $(p=0,00)$ mostrando evidência de dependência estatística, ao contrário do fato realizado pelo Pai, $(\mathrm{p}=0,16)$ (Tabela 3$)$. 
Tabela 3. Violência segundo faixa etária e autor da agressão provável. São José do Rio Preto, SP, 2015.

\begin{tabular}{|c|c|c|c|c|c|c|c|c|c|c|c|}
\hline \multirow{2}{*}{$\begin{array}{c}\text { Informação } \\
\text { Pai }\end{array}$} & \multicolumn{2}{|c|}{0 a 9 anos } & \multicolumn{2}{|c|}{10 a 19 anos } & \multicolumn{2}{|c|}{20 a 39 anos } & \multicolumn{2}{|c|}{$\begin{array}{l}40 \text { anos ou } \\
\text { mais }\end{array}$} & \multicolumn{2}{|l|}{ Total } & \multirow[t]{2}{*}{$\begin{array}{c}\text { Valor } \\
\mathbf{p}\end{array}$} \\
\hline & $\mathbf{N}$ & $\%$ & $\mathbf{N}$ & $\%$ & $\mathbf{N}$ & $\%$ & $\mathbf{N}$ & $\%$ & $\mathbf{N}$ & $\%$ & \\
\hline Sim & 139 & 31,4 & 49 & 15,0 & 5 & 0,9 & 0 & 0,0 & 193 & 11,4 & \\
\hline Não & 286 & 64,7 & 268 & 82,0 & 530 & 97,6 & 374 & 96,9 & 1458 & 85,9 & \\
\hline Não se Aplica & 17 & 3,8 & 10 & 3,1 & 8 & 1,5 & 12 & 3,1 & 47 & 2,8 & \\
\hline Ignorado & 0 & 0,0 & 0 & 0,0 & 0 & 0,0 & 0 & 0,0 & 0 & 0,0 & \\
\hline TOTAL & 442 & 100,0 & 327 & 100,0 & 543 & 100,0 & 386 & 100,0 & 1698 & 100,0 & 0,160 \\
\hline Mãe & $\mathbf{N}$ & $\%$ & $\mathbf{N}$ & $\%$ & $\mathbf{N}$ & $\%$ & $\mathbf{N}$ & $\%$ & $\mathbf{N}$ & $\%$ & \\
\hline Sim & 304 & 68,8 & 93 & 28,4 & 3 & 0,6 & 4 & 1,0 & 404 & 23,8 & \\
\hline Não & 130 & 29,4 & 227 & 69,4 & 532 & 98,0 & 371 & 96,1 & 1260 & 74,2 & \\
\hline Não se aplica & 8 & 1,8 & 7 & 2,1 & 8 & 1,5 & 11 & 2,8 & 34 & 2,0 & \\
\hline Ignorado & 0 & 0,0 & 0 & 0,0 & 0 & 0,0 & 0 & 0,0 & 0 & 0,0 & \\
\hline TOTAL & 442 & 100,0 & 327 & 100,0 & 543 & 100,0 & 386 & 100,0 & 1698 & 100,0 & 0,000 \\
\hline Cônjuge & $\mathbf{N}$ & $\%$ & $\mathbf{N}$ & $\%$ & $\mathbf{N}$ & $\%$ & $\mathbf{N}$ & $\%$ & $\mathbf{N}$ & $\%$ & \\
\hline Sim & 2 & 0,5 & 9 & 2,8 & 138 & 25,4 & 57 & 14,8 & 206 & 12,1 & \\
\hline Não & 429 & 97,1 & 310 & 94,8 & 398 & 73,3 & 316 & 81,9 & 1453 & 85,6 & \\
\hline Ignorado & 11 & 2,5 & 8 & 2,4 & 7 & 1,3 & 13 & 3,4 & 39 & 2,3 & \\
\hline TOTAL & 442 & 100,0 & 327 & 100,0 & 543 & 100,0 & 386 & 100,0 & 1698 & 100,0 & 0,000 \\
\hline Própria Pessoa & $\mathbf{N}$ & $\%$ & $\mathbf{N}$ & $\%$ & $\mathbf{N}$ & $\%$ & $\mathbf{N}$ & $\%$ & $\mathbf{N}$ & $\%$ & \\
\hline Sim & 6 & 1,4 & 89 & 27,2 & 205 & 37,8 & 127 & 32,9 & 427 & 25,1 & \\
\hline Não & 425 & 96,2 & 232 & 70,9 & 334 & 61,5 & 250 & 64,8 & 1241 & 73,1 & \\
\hline Ignorado & 11 & 2,5 & 6 & 1,8 & 4 & 0,7 & 9 & 2,3 & 30 & 1,8 & \\
\hline TOTAL & 442 & 100,0 & 327 & 100,0 & 543 & 100,0 & 386 & 100,0 & 1698 & 100,0 & 0,007 \\
\hline Outros & $\mathbf{N}$ & $\%$ & $\mathbf{N}$ & $\%$ & $\mathbf{N}$ & $\%$ & $\mathbf{N}$ & $\%$ & $\mathbf{N}$ & $\%$ & \\
\hline Sim & 62 & 14,0 & 37 & 11,3 & 27 & 5,0 & 50 & 13,0 & 176 & 10,4 & \\
\hline Não & 368 & 83,3 & 280 & 85,6 & 505 & 93,0 & 324 & 83,9 & 1477 & 87,0 & \\
\hline Ignorado & 12 & 2,7 & 10 & 3,1 & 11 & 2,0 & 12 & 3,1 & 45 & 2,7 & \\
\hline TOTAL & 442 & 100,0 & 327 & 100,0 & 543 & 100,0 & 386 & 100,0 & 1698 & 100,0 & 0,942 \\
\hline
\end{tabular}

Um caso notificado pode ser encaminhado para mais de um serviço. Os percentuais foram calculados, considerandose o total de notificações 1.698. Fonte SINAN/Vigilância epidemiológica/ SMS SJRP/SP.

No encaminhamento das vítimas, $(84,2 \%)$ de 0 a 9 anos e $(61,8 \%)$ de 10 a 19 anos (crianças e adolescentes) foram encaminhadas, principalmente, para o
Conselho Tutelar. Para o atendimento à mulher, (44\%) de 20 a 39 anos foram para o atendimento à mulher e (52\%) Delegacia da Mulher, ao somarmos a faixa etária de 20 a 39 e 40 anos ou mais. Nas análises inferenciais, ao atingir $(p=0,00)$, fica evidente que o fator encaminhamento ao Conselho Tutelar está ligado diretamente à faixa etária, de acordo com os dados amostrais, conforme Tabela 4. 
Tabela 4. Casos notificados de violência, segundo encaminhamento e faixa etária. São José do Rio Preto, SP, 2015.

\begin{tabular}{|c|c|c|c|c|c|c|c|c|c|c|c|}
\hline \multirow{2}{*}{$\begin{array}{c}\text { Informação } \\
\text { Rede de Saúde }\end{array}$} & \multicolumn{2}{|c|}{$\begin{array}{l}0 \text { a } 9 \\
\text { anos }\end{array}$} & \multicolumn{2}{|c|}{10 a 19 anos } & \multicolumn{2}{|c|}{20 a 39 anos } & \multicolumn{2}{|c|}{$\begin{array}{l}40 \text { anos ou } \\
\text { mais }\end{array}$} & \multicolumn{2}{|l|}{ Total } & \multirow[t]{2}{*}{$\begin{array}{c}\text { Valor } \\
\mathbf{P}\end{array}$} \\
\hline & $\mathbf{N}$ & $\%$ & $\mathbf{N}$ & $\%$ & $\mathbf{N}$ & $\%$ & $\mathbf{N}$ & $\%$ & $\mathbf{N}$ & $\%$ & \\
\hline Sim & 28 & 6,3 & 56 & 17,1 & 74 & 13,6 & 33 & 8,5 & 191 & 11,2 & \\
\hline Não & 405 & 91,6 & 267 & 81,7 & 458 & 84,3 & 340 & 88,1 & 1470 & 86,6 & \\
\hline Ignorado & 9 & 2,0 & 4 & 1,2 & 11 & 2,0 & 13 & 3,4 & 37 & 2,2 & \\
\hline TOTAL & 442 & 100,0 & 327 & 100,0 & 543 & 100,0 & 386 & 100,0 & 1698 & 100,0 & 0,143 \\
\hline $\begin{array}{l}\text { Assistência } \\
\text { Social }\end{array}$ & $\mathbf{N}$ & $\%$ & $\mathbf{N}$ & $\%$ & $\mathbf{N}$ & $\%$ & $\mathbf{N}$ & $\%$ & $\mathbf{N}$ & $\%$ & \\
\hline Sim & 10 & 2,3 & 18 & 5,5 & 44 & 8,1 & 59 & 15,3 & 131 & 7,7 & \\
\hline Não & 421 & 95,2 & 306 & 93,6 & 487 & 89,7 & 315 & 81,6 & 1529 & 90,0 & \\
\hline Ignorado & 11 & 2,5 & 3 & 0,9 & 12 & 2,2 & 12 & 3,1 & 38 & 2,2 & \\
\hline TOTAL & 442 & 100,0 & 327 & 100,0 & 543 & 100,0 & 386 & 100,0 & 1698 & 100,0 & 0,000 \\
\hline $\begin{array}{c}\text { Atendimento a } \\
\text { Mulher }\end{array}$ & $\mathbf{N}$ & $\%$ & $\mathbf{N}$ & $\%$ & $\mathbf{N}$ & $\%$ & $\mathbf{N}$ & $\%$ & $\mathbf{N}$ & $\%$ & \\
\hline Sim & 5 & 1,1 & 29 & 8,9 & 239 & 44,0 & 140 & 36,3 & 413 & 24,3 & \\
\hline Não & 426 & 96,4 & 294 & 89,9 & 293 & 54,0 & 234 & 60,6 & 1247 & 73,4 & \\
\hline Ignorado & 11 & 2,5 & 4 & 1,2 & 11 & 2,0 & 12 & 3,1 & 38 & 2,2 & \\
\hline TOTAL & 442 & 100,0 & 327 & 100,0 & 543 & 100,0 & 386 & 100,0 & 1698 & 100,0 & 0,000 \\
\hline $\begin{array}{l}\text { Conselho } \\
\text { Tutelar }\end{array}$ & $\mathbf{N}$ & $\%$ & $\mathbf{N}$ & $\%$ & $\mathbf{N}$ & $\%$ & $\mathbf{N}$ & $\%$ & $\mathbf{N}$ & $\%$ & \\
\hline Sim & 372 & 84,2 & 202 & 61,8 & 8 & 1,5 & 5 & 1,3 & 587 & 34,6 & \\
\hline Não & 62 & 14,0 & 121 & 37,0 & 528 & 97,2 & 370 & 95,9 & 1081 & 63,7 & \\
\hline Ignorado & 8 & 1,8 & 4 & 1,2 & 7 & 1,3 & 11 & 2,8 & 30 & 1,8 & \\
\hline TOTAL & 442 & 100,0 & 327 & 100,0 & 543 & 100,0 & 386 & 100,0 & 1698 & 100,0 & 0,000 \\
\hline $\begin{array}{l}\text { Delegacia da } \\
\text { Mulher }\end{array}$ & $\mathbf{N}$ & $\%$ & $\mathbf{N}$ & $\%$ & $\mathbf{N}$ & $\%$ & $\mathbf{N}$ & $\%$ & $\mathbf{N}$ & $\%$ & \\
\hline Sim & 4 & 0,9 & 32 & 9,8 & 171 & 31,5 & 81 & 21,0 & 288 & 17,0 & \\
\hline Não & 427 & 96,6 & 291 & 89,0 & 366 & 67,4 & 294 & 76,2 & 1378 & 81,2 & \\
\hline Ignorado & 11 & 2,5 & 4 & 1,2 & 6 & 1,1 & 11 & 2,8 & 32 & 1,9 & \\
\hline TOTAL & 442 & 100,0 & 327 & 100,0 & 543 & 100,0 & 386 & 100,0 & 1698 & 100,0 & 0,000 \\
\hline
\end{tabular}

\section{DISCUSSÃo}

A violência contra a mulher é um tema que tem atraído à atenção de governantes, estudiosos, bem como da sociedade geral em virtude do número de casos crescentes. Para diminuir tal agravante, iniciativas e ações, realizadas mundo afora, demonstram uma crescente tendência no reconhecimento da relevância dessa forma de violência como problema e objeto de intervenções da Saúde Pública. A construção da violência no âmbito doméstico está historicamente vinculada à posição que homens e mulheres devem assumir na sociedade. De acordo com a cultura androcêntrica, ainda hoje legitimada pela sociedade, os homens assumem o lugar de autoridade máxima, seja na situação de pai ou de esposo ${ }^{9-11}$.

A síndrome do trauma pós-violação, apontada como um agravo decorrente das variadas formas de violência impetradas contra a mulher se caracteriza como uma fase aguda de desorganização no estilo de vida da pessoa agredida e de sua família, demandando um processo prolongado de reorganização comportamental. Os sinais e os sintomas camuflam um silêncio que demonstra sentimentos de humilhação, vergonha e revolta ${ }^{12}$.

Após a Lei Maria da Penha, ter sido sancionada, o número de casos de mulheres com demanda de exame de corpo de delito no Instituto Médico Legal (IML) teria diminuído 
consideravelmente, questionando se a Lei teria sido forte o suficiente para inibir os agressores ou se teria contribuído para um maior silenciamento das mulheres. Outra hipótese estaria associada ao fato de muitas mulheres terem sido educadas para suportarem silenciosamente as opressões, na tentativa de manterem o modelo idealizado de relacionamento ou para garantir o equilíbrio, a sustentação e a manutenção da família' ${ }^{12,13}$.

Muitas vítimas procuram alternativas à denúncia formal, como a ajuda de parentes, de amigos e da Igreja, e não fazem nada a respeito da agressão sofrida, sendo o principal motivo o medo do agressor, seguido de dependência financeira e a preocupação com a criação dos filhos. No entanto, a magnitude do problema é bem maior, pois o silêncio de muitas vítimas faz com que inúmeros casos não sejam denunciados, mascarando os dados epidemiológicos ${ }^{14-15}$.

A violência contra a mulher perpetua no decorrer da história. Tem sua origem no núcleo familiar mediado e regido pelo patriarca, o qual atribui aos homens a autoridade sobre a mulher, subjugando-a, dando-lhes a condição, em algumas ocasiões, atingir os limites da violência. A Lei Maria da Penha coíbe a incidência dos casos de violência doméstica e familiar contra mulher, porém apesar deste artifício legal, a vitimização de mulheres é crescente ${ }^{16-18}$.

$\mathrm{Na}$ maioria dos casos, a mulher é economicamente dependente do agressor, por isto, é comum que não notifiquem a agressão. Para tanto, os principais motivos para que as vítimas se silenciem frente à violência são o risco de uma punição, dependência econômica, de manter-se sozinha, consideração de que permanecer com o agressor será melhor para os filhos, preconceitos e estereótipos de gênero, familiares ou culturais ${ }^{19}$.

A família é a base da sociedade, porém no contexto de violência, ao invés de oferecer apoio e segurança, ausenta-se de sua responsabilidade e priva a criança e o adolescente de seus direitos, prejudicando seu estado de desenvolvimento nas esferas física, social, comportamental, emocional e cognitiva. Os principais motivos para a ocorrência desse tipo de violência são baixa renda familiar, baixo nível de instrução, pobreza e falta de planejamento familiar ${ }^{20}$.

Em relação aos adolescentes, a violência física praticada pelos pais relaciona-se frequentemente à alteração comportamental dos filhos e também à fraqueza dos laços afetivos, podendo esses laços, estar fragilizados desde a infância, refletindo em apatia entre pais e filhos na adolescência. Os perpetradores, na tentativa de disciplinar, utilizam da força física tornando a criança e adolescente objeto de maus tratos ${ }^{21}$.

No presente estudo, a mãe foi a maior responsável pelos casos de negligência, violência física e abandono. Este fato pode estar relacionado à situação da sociedade pósmoderna que tem determinado significativo aumento no contingente de mulheres chefes de família, com respectiva degradação da qualidade de vida e aumento da pobreza.

Nesse contexto, é de se esperar que condições de vida subalternas possam traduzir, além da negligência contra crianças, negligência e violência social vivenciada também pelas mulheres (mães) que não dispõem de condições singulares, particulares e estruturais para uma vida digna para si mesma e, consequentemente, para seus filhos. Historicamente, à mulher tem sido atribuído o papel de cuidadora. Mesmo quando ela assume o trabalho no espaço público, o trabalho doméstico e do cuidado dos filhos não deixa de ser atribuição feminina ${ }^{22,23}$.

No Brasil, a violência contra crianças e adolescentes é um problema relevante e ainda muito pouco pesquisado de forma aprofundada, para compreender tal fenômeno sobre os vários prismas associados e permitir ações de prevenção e intervenção adequadas. Os estudiosos sobre o assunto de violência infanto-juvenil são unanimes em destacar a magnitude do problema e sobre a necessidade dessa realidade ser conhecida e reconhecida em suas diferentes nuances. Com isso e possível obter subsídios para fazer diagnósticos abrangentes acerca dessa problemática social e elaborar, propor e implementar estratégias de intervenção e de prevenção de sua ocorrência e, dos agravos que dela resultam ${ }^{24}$. 
São de suma importância que sejam discutidas e elencadas estratégias que visem a família como foco de intervenção. Estudos internacionais, frisam a relevância da implementação de programas no combate a violência na infância, com enfoque nas atividades que proporcionem o aprimorar de competências para a fase adulta, como estratégias que promovem o fortalecimento de vinculo familiar, excluindo do convívio atitudes violentas ${ }^{25,26}$.

Tais fatores podem refletir na queda da incidência de violência na vida adulta, principalmente impedindo futuros ciclos de reprodução da violência contra crianças, já que, vínculos violentos intrafamiliares aumentam a probabilidade de ações violentas na infância e na fase de vida adulta ${ }^{25,26}$.

Um estudo ${ }^{27}$ sobre análise da percepção de vários profissionais estudados revelou despreparo em relação à temática de violência contra crianças e adolescentes. Além disso, comprovou que os enfermeiros das unidades pediátricas pesquisadas devem assumir o compromisso técnico-científico, social, político e moral, no atendimento a crianças e adolescentes vítimas de violências, assim como devem cumprir seu papel na capacitação dos auxiliares e técnicos de enfermagem no contexto da atenção a vítimas infanto-juvenis em situações de maus tratos físicos. 0 conhecimento da legislação e a atuação efetiva permitem minimizar a subnotificação, fato que mascara a real situação da violência ${ }^{27}$.

Neste estudo a própria pessoa $(70,7 \%)$ na faixa etária 20 a 39 anos e 40 anos ou mais foi o principal perpetuador da agressão. Analisar a periculosidade de uma violência autoprovocada tende a ser uma tarefa complexa, pois são ações planejadas que não foram concluídas, e é possível que algumas dessas tentativas não tenham o propósito de conclusão, porém, nenhum caso deve ser negligenciado, pois dos que intentam contra a própria vida $(20 \%)$ a $(30 \%)$ recidivam na tentativa, e destes, (10\%) obtém como desfecho o óbito ${ }^{15,18}$.

Nem todos os casos de suicídio podem ser prevenidos, entretanto muitas vidas serão salvas se todas as pessoas que tentaram o suicídio fossem adequadamente abordadas e tratadas. 0 impacto da violência praticada por parceiro íntimo representa risco ao suicídio três vezes maior entre as mulheres que sofreram violência física moderada e oito vezes maior entre as que sofreram violência física grave, quando comparadas com as que não sofreram violência física ${ }^{19,28}$.

É notória a importância de implementação de políticas públicas que preencham as lacunas das redes de apoios para o encaminhamento das vítimas de violência com enfoque na qualificação e melhores condições de trabalho para os profissionais em questão, assim como para os usuários além de fortalecer as perspectivas de rede para garantir o acompanhamento dos grupos de risco. As variadas maneiras de agressão maculam os direitos humanos e confirmam a importância de uma eficiente rede de apoio, com enfoque interdisciplinar e multidisciplinar, agregando profissionais qualificados para reconhecer os casos de violência, conduzir e ou encaminhar conforme a situação, contribuindo significativamente no processo de saúde-doença das vítimas de violência ${ }^{23,24}$.

Os profissionais de saúde são identificados como peças-chave para o reconhecimento dos casos de violência, já que as vítimas que a vivenciam, tendem a procurar os serviços, em decorrência das consequências físicas e psicológicas que sofrem. Além do reconhecimento dos casos, o profissional deve ser capacitado para trabalhar as questões de gênero e a construção de autonomia, auxiliando na prevenção de novos casos. Os incrementos das produções científicas podem contribuir para o reconhecimento das estratégias, das limitações e das potencialidades, com vistas à redução das desigualdades de gênero e das vítimas de violência ${ }^{15,29,30}$.

A intersetorialidade das ações e a formação de redes para atendimento às pessoas em situação de violência são indispensáveis para a condução de ações de promoção da saúde e prevenção de agravos.

Espera-se, com esta caracterização dos casos de violência na região pesquisada, possa se incentivar a notificação e contribuir para a 
maior divulgação de informações, além de continuar com o processo de articulação e organização entre todas as redes de apoio do município para que possam subsidiar a elaboração de políticas públicas integradas e intersetoriais, dirigindo-se a linha de cuidado por tipos de violências e nas diferentes faixas etárias para que efetivamente promovam a saúde e a qualidade de vida dessas vítimas.

\section{CONCLUSÃO}

Conclui-se que apesar de na região pesquisada estar sendo realizadas, as notificações de violência, esta ocorre de forma fragmentada, apenas as redes de saúde, o conselho tutelar e o centro de referência à saúde da mulher realizam a notificação.

Cabe ao Estado juntamente com município propor que todos os órgãos de apoio participem de forma assídua na notificação de violências, para propor medidas de ações e controle, para que os dados epidemiológicos do município não ficam subnotificados.

Os resultados apresentados apontam a necessidade de continuar o processo de articulação e organização entre as redes de apoio, além de mais pesquisas sobre a problemática, destacando as questões das violências por ciclos de vida para elaboração de planejamentos estratégicos como a linha de cuidados para realização de ações de prevenção e controle para melhorar a notificação, o esclarecimento e o acolhimento ao enfrentamento das vítimas de violência interpessoal/autoprovocadas do Município.

\section{REFERÊNCIAS}

1. Dahlberg LL, Krug EG. Violência: um problema global de saúde pública. Ciênc Saúde Colet. [internet] 2007; [citado em 10 jul 2016]; 11(Suppl):1163-78.

DOI:

http://dx.doi.org/10.1590/S1413-

81232006000500007

2. Krug EG, Dahlberg LL, Mercy JA, Zwi AB, Lozano $\mathrm{R}$, editores. World report on violence and health [Internet]. Geneva: WHO; 2002 [citado em 10 jul 2016]. Disponível em: http://www.who.int/violence_injury_preventio n/violence/world_report/en/full_en.pdf

3. Ministério da Saúde (Br). Saúde Brasil 2013: uma análise da situação de saúde e das doenças transmissíveis relacionadas à pobreza. Brasília, DF: Ministério da Saúde; 2014. 386p.

4. Ministério da Saúde $(\mathrm{Br})$, Secretaria de Vigilância em Saúde. Viva: instrutivo notificação de violência interpessoal e autoprovocada. 2 ed. Brasília, DF: Ministério da Saúde; 2016. 92p.

5. Presidência da República (Brasil). Lei n. 8.069, de 13 de julho de 1990. Dispõe sobre o Estatuto da Criança e do Adolescente e dá outras providências. D.O.U., 16 jul 1990. Seção 1:1356.

6. Câmara dos Deputados (Brasil). Projeto de Lei 4.552-A, de 25 de fevereiro de 2016. Complementa a Lei $n^{\circ} 10.778$, de 24 de novembro de 2003, que estabelece a notificação compulsória, no território nacional, do caso de violência contra a mulher que for atendida em serviços de saúde públicos ou privados [Internet]. Brasília, DF: Câmara dos Deputados; 2017 [citado em 20 mai 2018]. Disponível em: http://www.camara.gov.br/proposicoesWeb/p rop_mostrarintegra;jsessionid $=907 \mathrm{EC} 33223 \mathrm{C} 7$ 769CBCDED1326B0E3424.proposicoesWebExt erno1? codteor $=1580989 \&$ filename $=$ Avulso+$\mathrm{PL}+4552 / 2016$

7. Presidência da República (Brasil). Lei $\mathrm{n}^{\mathrm{o}}$ 10.741, de 1 o de outubro de 2003. Dispõe sobre o Estatuto do Idoso e dá outras providências [Internet]. D.O.U., Brasília, DF, 3 out 2003 [citado em 3 nov 2016]. Disponível em: http://www.planalto.gov.br/CCivil_03/leis/20 03/L10.741.htm

8. Ministério da Saúde (Br). Portaria n. 2.836, de $1^{\circ}$ de dezembro de 2011. Institui, no âmbito do Sistema Único de Saúde (SUS), a Política Nacional de Saúde Integral de Lésbicas, Gays, Bissexuais, Travestis e Transexuais [Internet]. Brasília, DF: Ministério da Saúde; 2011 [citado em 10 jul 2016]. Disponível: http://bvsms.saude.gov.br/bvs/saudelegis/gm /2011/prt2836_01_12_2011.html

9. Vieira EM, Perdona GSC, Santos MA. Fatores associados à violência física por parceiro íntimo em usuárias de serviços de saúde. Rev Saúde Pública [Internet]. 2011 [citado em 04 maio 2018]; 45(4):730-7. DOI: http://dx.doi.org/10.1590/S0034-

89102011005000034

10. Duarte MC, Fonseca RMGS, Souza V, Pena ED. Gênero e violência contra a mulher na literatura de enfermagem: uma revisão. Rev Bras Enferm. [Internet]. 2015 [citado em 04 maio 2018]; 68(2):325-32.

DOI: http://dx.doi.org/10.1590/0034

7167.2015680220i 
11. Lindner SR, Coelho EBS, Bolsoni CC, Rojas PF, Boing AF. Prevalência de violência física por parceiro íntimo em homens e mulheres de Florianópolis, Santa Catarina, Brasil: estudo de base populacional. Cad Saúde Pública [Internet]. 2015 [citado em 04 maio 2018]; 31(4):815-26. DOI: http://dx.doi.org/10.1590/0102-

\section{X00159913}

12. Martín-Baena D, Montero-Piñar I, EscribàAgüir V, Vives-Cases C. Violence against young women attending primary care services in Spain: prevalence and health consequences. Fam Pract. [Internet]. 2015 [citado em 10 jul 2018]; 32(4):381-6. DOI: https://doi.org/10.1093/fampra/cmv017 13. Acosta DF, Gomes VLO, Fonseca AD, Gomes GC. Violência contra a mulher por parceiro íntimo: (in)visibilidade do problema. Texto \& Contexto Enferm. [Internet] 2015 [citado em 10 set 2016]; 24(1):121-7. DOI: http://dx.doi.org/10.1590/010407072015001770013

14. Garcia LP, Duarte EC, Freitas LRS, Silva GDM. Violência doméstica e familiar contra a mulher: estudo de casos e controles com vítimas atendidas em serviços de urgência e emergência. Cad Saúde Pública [Internet]. 2016 [citado em 10 set 2016]; 32(4): e00011415. DOI: http://dx.doi.org/10.1590/0102311X00011415

15. Souza MJ, Silva JP, Barros LA, França AMB, Moura MRW. Caracterização dos casos de violência sexual contra a mulher em uma unidade de referência. Ciênc Biol Saúde [Internet]. 2014 [citado em 10 set 2016]; 2(1):177-92. Disponível em: https://periodicos.set.edu.br/index.php/fitsbio saude/article/view/1367/773

16. Presidência da República (Brasil). Lei $\mathrm{n}^{\mathrm{o}}$ 11.340, de 7 de Agosto de 2006. Cria mecanismos para coibir a violência doméstica e familiar contra a mulher, nos termos do $\S 8^{\circ}$ do art. 226 da Constituição Federal, da Convenção sobre a Eliminação de Todas as Formas de Discriminação contra as Mulheres e da Convenção Interamericana para Prevenir, Punir e Erradicar a Violência contra a Mulher; dispõe sobre a criação dos Juizados de Violência Doméstica e Familiar contra a Mulher; altera o Código de Processo Penal, o Código Penal e a Lei de Execução Penal; e dá outras providências [Internet]. D.O.U., Brasília, DF, 8 ago 2006 [citado em 10 set 2016]. Disponível em: www.planalto.gov.br/ccivil_03/_ato20042006/2006/lei/l11340.htm

17. Adeodato VG, Carvalho RR, Siqueira VR, Souza FGM. Qualidade de vida e depressão em mulheres vítimas de seus parceiros. Rev Saúde Publica [Internet]. 2005 [citado em 19 set 2016]; 39(1):108-13. doi: http://dx.doi.org/10.1590/S0034-

89102005000100014

18. Sinimbu RB, Mascarenhas MDM, Silva MMA, Carvalho MGO, Santos MR, Freitas MG. Caracterização das vítimas de violência doméstica, sexual e/ou outras violências no Brasil 2014. Saúde Foco [Internet]. 2016 [citado em 10 set 2016]; 1(1):[1-14]. Disponível em: https://smsrio.org/revista/index.php/revsf/ar ticle/view/199/178

19. Silva LEL, Oliveira MLC. Características epidemiológicas da violência contra a mulher no Distrito Federal, 2009 a 2012. Epidemiol Serv Saúde [Internet]. 2016 [citado em 19 set 2016]; 25(2):331-42. Disponível em: http://www.scielo.br/pdf/ress/v25n2/22379622-ress-25-02-00331.pdf

20. Zanelatto PF, Medeiros M, Santos WS, Bouttelet MD. Violência contra crianças e adolescentes: significados e atitudes por equipes da estratégia saúde da família. Ciênc Enferm. [Internet]. 2012 [citado em 10 jul 2016]; 18(2):41-9.

DOI:http://dx.doi.org/10.4067/S0717-

95532012000200005

21. Trabbold VLM, Caleiro RCL, Cunha CF, Guerra AMC. Concepções sobre adolescentes em situação de violência sexual. Psicol Soc. [Internet]. 2016 [citado em 10 set 2016]; 28(1):74-83.

DOI:http://dx.doi.org/10.1590/1807-

03102015v28n1p074

22. Oliveira EM, Felix TA, Mendonça CBL, Lima PSF, Freire AS, Moreira RMM. Aspectos epidemiológicos e o cuidado de enfermagem na tentativa de suicídio. Rev Enferm Contemp. [Internet]. 2016 [citado em 10 set 2016]; 5(2):184-92.

DOI:

http://dx.doi.org/10.17267/2317-

3378rec.v5i2.967

23. Almeida SA, Guedes PMM, Nogueira JA, França UM, Silva ACO. Investigação de risco para tentativa de suicídio em hospital de João Pessoa - PB. Rev Eletrônica Enferm. [Internet]. 2009 [citado em 10 set 2016]; 11(2):383-9. Disponível 
http://www.fen.ufg.br/revista/v11/n2/v11n2 a20.htm

24. Ferreira DLM, Soler ZASG. O que tem sido divulgado em periódicos científicos online no Brasil sobre violência contra crianças e adolescentes. Enferm Brasil. 2013; 12(2):112-9. 25. Freitas RJM, Moura NA, Monteiro ARM. Violência contra crianças/adolescentes em sofrimento psíquico e cuidado de enfermagem: reflexões da fenomenologia social. Rev Gaúch Enferm. [Internet]. 2016 [citado em 19 set 2016]; 37(1):e52887. DOI: http://dx.doi.org/10.1590/1983-

1447.2016.01.52887

26. Apostólico MR, Nóbrega CR, Guedes RN, Fonseca RMGS, Egry EY. Characteristics of violence against children in a Brazilian Capital. Rev Latinoam Enferm. [Internet]. 2012 [citado em 10 jul 2016]; 20(2):266-73. DOI: http://dx.doi.org/10.1590/S010411692012000200008

27. Pinto EC, Ferreira DLM, Soler ZASG. Physical abuse against children and teens: perception of pediatric nursing team. Rev Enferm UFPE on line. [Internet]. 2013 [citado em 04 maio 2018]; 7(6):4411-20. Disponível em: https://periodicos.ufpe.br/revistas/revistaenf ermagem/article/view/11681

28. Almeida LCG, Diniz NMF. Violência sexual: desvelando a realidade que acomete as mulheres. Rev Enferm UERJ. [Internet]. 2004 [citado em 04 maio 2018]; 12(1):88-94. Disponível em: http://www.facenf.uerj.br/v12n1/v12n1a15.p df

29. Garcia LP, Freitas LRS, Höfelmann DA. Avaliação do impacto da Lei Maria da Penha sobre a mortalidade de mulheres por agressões no Brasil, 2001-2011. Epidemiol Serv Saúde [Internet]. 2013 [citado em 04 maio 2018]; 22(3):383-94. Disponível em: http://scielo.iec.gov.br/pdf/ess/v22n3/v22n3 a03.pdf

30. Signorelli MC, Taft A, Pereira PPG. Intimate partner violence against women and healthcare in Australia: charting the scene. Ciênc Saúde Colet. [Internet]. 2012 [citado em 04 maio 2018]; 17(4):1037-48. DOI: http://dx.doi.org/10.1590/S141381232012000400025

CONTRIBUIÇÕES
Ana Lígia Fernandes Reis e Gabriela
Martins Espolador realizaram levantamento
dos dados e referências. Sônia Aparecida da
Cruz Oliveira colaborou no desenho do
estudo, revisão, interpretação dos dados e
discussão. Alexandre Lins Werneck
participou na discussão e revisão crítica.

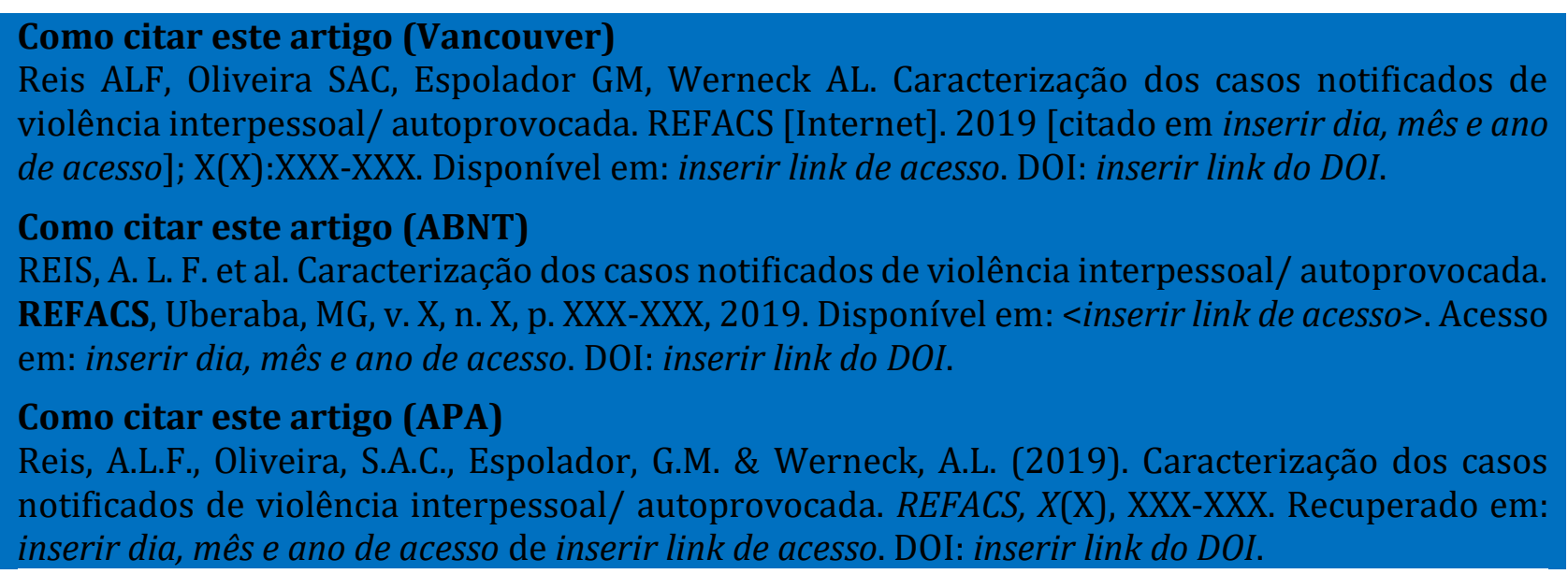

